\title{
Brief
}

\section{Prolonged survival of fully allogeneic cardiac grafts in naive mice and those with sensitization induced by antigen delivery through the respiratory tract}

\author{
Osamu Aramaki, MD, ${ }^{a}$ Nozomu Shirasugi, MD, ${ }^{\mathrm{b}}$ Yosinobu Akiyama, MD, ${ }^{\mathrm{b}}$ Yoshifumi Ikeda, MD, ${ }^{\mathrm{b}}$ Shiho Yabuki, MD, \\ Motohide Shimazu, MD, ${ }^{c}$ Masaki Kitajima, MD, ${ }^{c}$ and Masanori Niimi, MD, PhD, ${ }^{\mathrm{b}}$ Tokyo, Japan
}

From the Department of Surgery, ${ }^{a}$ Nihon University, the Department of Surgery, ${ }^{\mathrm{b}}$ Teikyo University, and the Department of Surgery, ${ }^{\mathrm{c}}$ Keio University, Tokyo, Japan.

Received for publication March 29, 2002; accepted for publication Jan 14, 2003.

Address for reprints: Masanori Niimi, MD, $\mathrm{PhD}$, Department of Surgery, Teikyo University, 2-11-1 Kaga, Itabashi-ku, Tokyo, Japan 173-8605 (E-mail: mniimi@med. teikyo-u.ac.jp).

J Thorac Cardiovasc Surg 2003;126:853-4 Copyright (C) 2003 by The American Association for Thoracic Surgery

$0022-5223 / 2003 \$ 30.00+0$

doi:10.1016/S0022-5223(03)00219-8

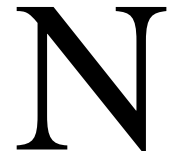

onresponsiveness to protein antigen induced by administration of the antigen through the alimentary or respiratory tract is well known as mucosal tolerance. ${ }^{1,2}$ We previously reported in this Journal that donor splenocytes delivered orally induce prolonged survival of fully allogeneic cardiac grafts and generate regulatory cells when delivered with a nondepleting anti-CD4 monoclonal antibody. ${ }^{3}$ Therefore, in this study we examined whether intratracheal delivery of alloantigen could induce prolonged survival of fully mismatched allogeneic cardiac grafts and regulatory cells.

\section{Methods and Results}

Mice were purchased from Sankyo (Sankyo Co, Ltd, Tokyo, Japan) and housed in conventional facilities of the Biomedical Services Unit at Teikyo Hospital and used between 8 and 12 weeks old in accordance with the Animal Care Guidelines of Teikyo University. Mouse hearts were transplanted into the abdomens of recipients by microsurgical technique. ${ }^{4}$

Naive CBA $\left(\mathrm{H} 2^{\mathrm{k}}\right)$ mice rejected fully allogeneic C57BL/10 $\left(\mathrm{H} 2^{\mathrm{b}}\right)$ cardiac grafts with a median survival of 12 days (group 1; Table 1). When CBA mice were pretreated with intratracheal delivery of $1 \times 10^{7} \mathrm{C} 57 \mathrm{BL} / 10$ splenocytes 7 days before transplantation of a C57BL/10 heart, all grafts demonstrated prolonged survival for longer than 50 days without any further immunosuppression (group 2 median survival 65 days, Table 1). However, either intravenous or oral delivery of splenocytes alone could not induce prolonged survival (groups 3 and 4 median survivals 13 and 16 days, respectively; Table 1), suggesting that the intratracheal route was the most effective in inducing hyporesponsiveness to alloantigen.

Next, adoptive transfer study was performed to examine the existence of regulatory cells. CBA mice were pretreated with intratracheal administration of $1 \times 10^{7}$ splenocytes. Seven days later, the mice were killed and $5 \times 10^{7}$ splenocytes were adoptively transferred into another set of naive CBA mice. C57BL/10 cardiac grafts were transplanted into the second recipients at the same day of the transfer. All C57BL/10 cardiac grafts had prolonged survival for longer than 50 days in the second CBA recipients when the first CBA mice were pretreated with C57BL/10 (group 5 median survival 64 days; Table 1) but not with either recipient-type CBA (graft survivals 10, 12, and 14 days, median survival 12 days) or third-party BALB/c (graft survivals 10, 12, and 12 days, median survival 12 days) splenocytes, suggesting that regulatory cells were generated 7 days after the pretreatment and were donor specific.

In clinical organ transplantation, the protocol would be more feasible when graft survival is prolonged even in sensitized recipients. Human recipients are thought to be sensitized by environmental antigens, which can be cross-reactive to alloantigen. When CBA mice were transplanted with C57BL/10 cardiac grafts 14 days after C57BL/10 skin grafts, rejection of C57BL/10 cardiac grafts was accelerated (group 6 median survival 7 days; Table 1) compared with that in naive CBA mice (group 1 median survival 12 days; Table 1). This demonstrated that skin grafting sensitized the CBA mice. When intratracheal delivery of C57BL/10 splenocytes was performed 7 days after skin grafting $(7$ days before transplantation of a 
TABLE 1. Survival of fully allogeneic C57BL/10 cardiac grafts in CBA recipients

\begin{tabular}{|c|c|c|c|c|c|}
\hline Group & Pretreatment & Recipient & Graft survival (d) & $\begin{array}{c}\text { Median } \\
\text { survival (d) }\end{array}$ & $P$ value* \\
\hline 1 & None & Naive & $9,11,12,14,14$ & 12 & \\
\hline 2 & Intratracheal delivery† & Naive & $50,54,60,65,70,82,94$ & 65 & $<.01 \ddagger$ \\
\hline 3 & Intravenous delivery§ & Naive & $12,13,13,13,18$ & 13 & NS $\ddagger$ \\
\hline 4 & Oral delivery\| & Naive & $12,12,16,16,16,16,18$ & 16 & NS $\ddagger$ \\
\hline 5 & Adoptive transfer? & Naive & $52,54,58,64,68,70,74$ & 64 & $<.01 \ddagger$ \\
\hline 6 & None & Sensitized ${ }^{* *}$ & $5,7,7,7,9$ & 7 & $<.01 \ddagger$ \\
\hline 7 & Intratracheal delivery† & Sensitized ${ }^{* *}$ & $10,10,14,14,23$ & 14 & $<.01 \ddagger \ddagger$ \\
\hline 8 & Adoptive transfer§ & Sensitized ${ }^{* *}$ & $9,11,15,21,26$ & 15 & $<.01 \ddagger \ddagger$ \\
\hline
\end{tabular}

NS, Not significant.

*B Mann-Whitney $U$ test.

$\dagger 1 \times 10^{7}$ C57BL/10 splenocytes were delivered intratracheally 7 days before cardiac grafting.

$\ddagger$ Versus group 1 .

$\S 1 \times 10^{7}$ C57BL/10 splenocytes were delivered intravenously 7 days before cardiac grafting.

$\| 1 \times 10^{7} \mathrm{C} 57 \mathrm{BL} / 10$ splenocytes were fed 7 days before cardiac grafting.

$\eta 5 \times 10^{7}$ C57BL/10 splenocytes from CBA mice pretreated with intratracheal delivery of $1 \times 10^{7}$ C57BL/10 splenocytes were adoptively transferred into naive or sensitized recipients on the same day of cardiac grafting.

${ }^{* *}$ CBA mice were transplanted with C57BL/10 skin 14 days before cardiac grafting.

††Versus group 6.

C57BL/10 heart), all C57BL/10 cardiac grafts showed modest but clearly significant prolonged survival in the sensitized CBA mice (group 7 median survival 14 days; Table 1). Moreover, when the sensitized mice received adoptive transfer of $5 \times 10^{7}$ splenocytes from naive CBA mice pretreated with intratracheal delivery of $1 \times$ $10^{7} \mathrm{C} 57 \mathrm{BL} / 10$ splenocytes, median survival of C57BL/10 cardiac grafts in the sensitized CBA mice was prolonged from 7 to 15 days (groups 6 and 8; Table 1). These results demonstrate that intratracheal delivery of alloantigen is also effective in inducing prolonged survival and generating regulatory cells in the sensitized mice and that the regulatory cells are effective against activated responder cells.

\section{Discussion}

We have previously shown that anti-CD4 in combination with oral delivery of alloantigen can induce indefinite survival of fully allogeneic cardiac grafts and generates regulatory cells. However, oral delivery without anti-CD4 induced slightly modest prolonged survival of a single MHC-mismatched graft but not fully allogeneic cardiac grafts. ${ }^{5}$ In this study, we clearly demonstrated the importance of the respiratory tract to induce hyporesponsiveness against fully allogeneic grafts. When the respiratory tract was chosen as the route of antigen delivery, hyporesponsiveness against fully mismatched alloantigens was induced without any further treatment. Moreover, the regulatory cells generated after intratracheal delivery of alloantigen were able to induce prolonged graft survival in not only naive but also sensitized recipients, suggesting that the regulatory cells can turn off memory cells. This observation can be applied in clinical organ transplantation to induce hyporesponsiveness against fully allogeneic cardiac grafts by means of the respiratory tract.

\section{References}

1. WeinerHL. Oral tolerance: immune mechanisms and treatment of autoimmune disease. Immunol Today. 1997;18:335-43.

2. ChenY, InobeJI, MarksR, GonnellaP, KuchrooVK, WeinerHL. Peripheral deletion of antigen-reactive T cell in oral tolerance. Nature. 1995; 376:177-80.

3. NiimiM, IkedaY, KanS, ShirasugiN, HamanoK. Indefinite survival of fully allogeneic cardiac grafts induced by antigen delivery through the alimentary tract. $J$ Thorac Cardiovasc Surg. 2001;122: 629-30.

4. NiimiM. The technique for heterotopic cardiac transplantation in mice: experience of 3000 operations by one surgeon. J Heart Lung Transplant. 2001;20:1123-8.

5. NiimiM, WitzkeO, BushellA, HaraM, MorrisPJ, WoodKJ. Nondepleting anti-CD4 monoclonal antibody enhances the ability of oral alloantigen delivery to induce indefinite survival of cardiac allografts: oral tolerance to alloantigen. Transplantation. 2000;70:1524-8. 